\title{
Die Problematik der Genera der Substantive in der Übersetzung von Märchen
}

\section{Problems of Translating Genders of Substantives in Fairy Tales}

\section{Markéta Šmalcová}

\begin{abstract}
In his book "Umění překladu" (1963), Jiří Levý describes three fundamental steps of the translation process. He calls for the thorough understanding, correct interpretation as well as stylistic transformation of the original text so that its meaning will not get lost as a result of inaccurate translation.

The paper deals with some of the interesting problems that arise when translating words for fairy tale characters and objects from "Kinder- und Hausmärchen" by the Brothers Grimm into Czech or Italian. In these languages the corresponding words frequently have different genders from German. So the paper addresses the question of how much of a challenge the gender differences in German, Czech and Italian can be for the translator.
\end{abstract}

\section{Keywords}

genders, fairy tales, Brothers Grimm, Italian, Czech, German, translation 


\section{Grundprinzipien der Übersetzung bei Jiří Levý}

Jiř́ Levý beschäftigt sich in seinem Werk ,Die Kunst der Übersetzung' mit der Methodologie der Übersetzung von Schöner Literatur. Nach seiner Untersuchung gibt es drei Phasen der Übersetzerarbeit, und zwar: Erfassen, Interpretieren und Umsetzen einer Vorlage (vgl. LEVÝ 1963: 53).

\subsection{Das Erfassen der Vorlage}

Ein guter Übersetzer muss zunächst ein guter Leser sein. Er muss vor allem am philologischen Erfassen des Textes arbeiten. Ebenso wichtig ist die grundsätzliche Untersuchung des historischen und gesellschaftlichen Kontextes. Ohne Sachkenntnisse und Kenntnis der Herkunft des Übersetzungstextes sollte kein Übersetzer arbeiten' (vgl. LEVÝ 1963: 53).

\subsection{Das Interpretieren der Vorlage}

Aufgrund der Verschiedenheit der Sprachen ist eine vollkommene Übereinstimmung von Original und Übersetzung nicht möglich. Der Übersetzer muss deswegen an manchen Stellen interpretieren, um dem Original gerecht zu werden. Sehr hilfreich sind dabei die objektiven Werte des Werkes und wesentliche Fakten, d.h. dass der Übersetzer auf Persönliches verzichtet und in die literarische Welt eintaucht, in die fiktionale Welt des Werks eintaucht (vgl. LEVÝ 1963: 57). Eine Deformierung des ursprünglichen Textes sollte dabei vermieden werden. Die einfühlsame Bearbeitung gilt als eines der Grundprinzipien der Übersetzung (vgl. LEVÝ 1963: 59).

\subsection{Das Umsetzen der Vorlage}

Da eine mechanische, also wörtliche Übersetzung eines künstlerischen Textes, wie bereits unter 1.2 erwähnt, nicht möglich ist, muss der Übersetzer die richtigen Formulierungen finden, um den Text nicht kalt, farblos und gefühllos erscheinen zu lassen. Gut ist es, dabei alle sprachliche Besonderheiten und Möglichkeiten der Zielsprache zu nutzen. Besonders gilt das bei der Übersetzung von gebundener Sprache im Allgemeinen und Poesie im Besonderen, aber grundsätzlich auch bei der Übersetzung von Diminutiven, Phraseologismen, Neologismen, Wortspielen u.a. (vgl. LEVÝ 1963: 68).

\section{Die Übersetzung von Genera}

Einen sehr interessanten Forschungsgegenstand stellen im Zusammenhang mit dem Übersetzen die Genera in verschiedenen Sprachsystemen dar. Bei der Übersetzung stößt 
man sicher darauf, dass sich die Sprachen in ihren grammatischen Systemen unterscheiden. Das häufigste Problem scheint die Übersetzung der Genera der Substantive zu sein. Da muss sich der Übersetzer entscheiden, ob er metaphorisch oder wörtlich übersetzen soll. (vgl. KONVIČKA, RAŠNEROVÁ, ZBORNÍKOVÁ 2017: 63/64) Und dass das nicht immer einfach ist, belegt schon der mittelalterliche Text Laudes Creaturarum. Es handelt sich um einen Lobgesang auf die Schönheit der Schöpfung. Dieser Text entstand vermutlich Ende 1224, und der Autor war kein anderer als der Hl. Franziskus von Assisi (vgl. WHITFIELD 1986: 33). Ins Deutsche wurden die Laudes Creaturarum als Der Sonnengesang (vgl. REINDERS 1986: 33) übersetzt. Im Tschechischen ist der Text als Píseñ tvorstva bekannt. Und vom Lateinischen ins Italienische wurde der Titel als Cantico delle creature (vgl. BERTIN 2016) übertragen.

Am interessantesten sind bei der Übersetzung dieses Werkes vor allem die Bezeichnungen der Sonne, des Mondes und des Todes, die vom Hl. Franziskus personifiziert werden und den Status Schwester oder Bruder erhalten.

\begin{tabular}{|l|l|l|l|l|l|l|l|}
\hline \multicolumn{9}{|c|}{ Laudes Creaturarum } \\
\hline \multicolumn{3}{|c|}{ Deutsch } & \multicolumn{4}{c|}{ Tschechisch } & \multicolumn{2}{|c|}{ Italienisch tvorstva } \\
\hline $\mathrm{f}$ & Sonner Sonnengesang & Bruder Sonne & $\mathrm{n}$ & slunce & bratr slunce & \multicolumn{2}{c|}{ Cantico delle Creature } \\
\hline $\mathrm{m}$ & Mond & Schwester Mond & $\mathrm{m} / \mathrm{f}$ & měsíc/luna & sestra luna & $\mathrm{f}$ & luna \\
\hline $\mathrm{m}$ & Tod & Schwester Tod & $\mathrm{f}$ & smrt & sestra smrt & $\mathrm{f}$ & morte \\
\hline
\end{tabular}

Tab. 1 Laudes Creaturarum

Tabelle 1 zeigt, wie sich die einzelnen Substantive, die im Sonnengesang vorkommen und die Bezeichnung Schwester oder Bruder haben, an die Genuszuordnung anpassen mussten. (Trotzdem haben sich einige Übersetzer ins Deutsche für „Schwester“ Sonne oder „Bruder“ Tod entschieden haben). Da sich die italienische Sprache aus dem Lateinischen entwickelte, wurde auch die Genuszuordnung behalten. Daher entstand bei der Übersetzung des lateinischen Textes ins Italienische kein Problem. Das trat erst auf, als Der Sonnengesang, ursprünglich in einer romanischen Sprache geschrieben, in eine germanische oder slawische Sprache übertragen werden sollte. Sollte man das grammatische Geschlecht bevorzugen oder bei der Übersetzung die Bedeutung und den Sinn der benutzten Bezeichnungen Bruder und Schwester (sore luna / Schwester Mond, frate sole / Bruder Sonne) berücksichtigen, auch wenn es natürlicher wäre, sich nach dem Genus in jener Sprache zu richten? Wenn man die deutschen, tschechischen und italienischen Ausdrücke dieses Liedes vergleicht, stellt man fest, dass die Bezeichnungen Bruder und Schwester in den Übersetzungen beibehalten wurden, obwohl die Sonne, der Mond und der Tod in allen drei Sprachen unterschiedliche Genera haben. Interessant ist die Tatsache, dass der Mond im Tschechischen sogar zwei Genera aufweist, je nachdem, ob wir eher den poetischen Ausdruck luna (eher poetischer Ausdruck) oder eher den neutralen Ausdruck měsíc wählen. Der größte Unterschied zeigt sich bei den Substantiven in der Tabelle beim Wort Sonne, das in jeder der erwähnten Sprachen ein anderes Genus aufweist. Diese Problematik spiegelt sich natürlich auch in anderen Texten wider, in denen 
dem Ausdruck eine soziale Bedeutung zugeschrieben wird, wie es zum Beispiel auch in Märchen geschieht.

\section{Die Genera im Deutschen, Tschechischen und Italienischen}

Sprachwissenschaftlichen Untersuchungen nach unterscheidet man grammatisches und natürliches Genus. Sowohl in der deutschen als auch in der italienischen und tschechischen Sprache findet man interessante Ausnahmen und Sprachkuriositäten, die die einzelnen Sprachsysteme bei der Genuszuordnung aufweisen. Um zu zeigen, was für ein breites Spektrum von Merkwürdigkeiten einzelne Sprachen anbieten, werden die folgenden wenigen Beispiele aus der deutschen, italienischen und tschechischen Sprache angeführt. Bewusst wurden solche Substantive als Beispiele benutzt, die auch oft in Märchen vorkommen.

\subsection{Das Genus in der deutschen Sprache}

Im Deutschen gibt es drei Genera - Femininum, Maskulinum und Neutrum. In der deutschen Sprache übernehmen die zusammengesetzten Ausdrücke das Genus des letzten Nomens, z. B. die Bettelfrau. Die Diminutiva im Deutschen sind automatisch Neutra, die meistens auf

-chen oder -lein enden, so etwa bei Dornröschen, Hähnchen und Hühnchen (vgl. J. UND W. GRIMM 2010). Der deutsche Wortschatz umfasst auch Nomen, die die gleiche Lautung, doch auf Grund des unterschiedlichen grammatischen Geschlechts verschiedene Bedeutungen haben:

- die Taube (ein Vogel, eine taube Frau) x der Taube (ein tauber Mann) oder

- der Junge (Kind männlichen Geschlechts) x das Junge (ein junges Tier) (URL1).

\subsection{Das Genus in der tschechischen Sprache}

Im Tschechischen gibt es wie im Deutschen drei Genera - Femininum, Maskulinum und Neutrum. Die Maskulina werden zudem nach Belebtheit und Unbelebtheit unterschieden. Bei einigen Substantiven sind (wie auch in anderen Sprachen) zwei Genera möglich, ohne dass sich die Bedeutung des Substantives ändert, z.B. kedluben x kedlubna (Kohlrabi), okurka $x$ okurek (Gurke). Wie im Deutschen gibt es auch Abweichungen des grammatischen vom natürlichen Geschlecht: sirotek ( die Waise), chudák (der Arme), lenoch (der Faulenzer) (vgl. ŠTÍCHA 2010: 584), vnouče (das Enkelkind). Zu dieser Gruppe gehören auch die meisten Tiere: moucha (die Fliege), ryba (der Fisch), ježek (der Igel). Ab und zu findet man auch in der tschechischen Sprache auch witzige weibliche Formen, die durch Analogiebildung aus einer männlichen entstanden sind: krab (dt. Bed.) x krabice (Kiste), had (dt. Bed.)x hadice (Schlauch), lenoch x lenoška (Sofa in unseren Wörterbüchern „Lehnstuhl“). 
Im Gegensatz dazu werden Genera wie das von chot' (Ehefrau/Ehemann), nešika (Tolpatsch), naivka (Einfaltspinsel) erst durch die Deklination und/oder die kontextuelle Einbettung erkannt. (URL2).

\subsection{Das Genus in der italienischen Sprache}

Im Italienischen gibt es nur zwei Genera - Maskulinum und Femininum. Bei Substantiven, die etwas Belebtes bezeichnen, entspricht meistens das grammatische Genus dem natürlichen Geschlecht (vgl. HAMPLOVÁ 2004: 58):

il gatto (m) x la gatta (f) (Kater, Katze). Bei Substantiven, die etwas Nichtbelebtes bezeichnen, hängt die Bestimmung des Genus von linguistischen Bedingungen ab: fiaba, casa (f) (Märchen, Haus), ricordo, sole (m) (Erinnerung, Sonne). Einige Substantive im Italienischen haben sowohl für Feminina als auch für Maskulina dieselbe substantivische Form. Um die weibliche Form hervorzuheben, muss man einen weiblichen oder männlichen Artikel oder ein Adjektiv benutzen insegnante, giornalista, amante (Lehrer/in, Journalist/in, Liebhaber/in) (vgl. HAMPLOVÁ 2004: 59).

Einige Substantive haben nur eine Form für beide Genera. Bei diesen kann man nur durch eine zusätzliche Bezeichnung zwischen männlich oder weiblich unterscheiden. Das ist oft bei Tierbezeichnungen der Fall: tigre maschio (Tiger männlichen Geschlechts), medico-donna ( $\ddot{r} r z t i n)$.

Sowohl im Deutschen als auch im Italienischen gibt es Wörter, die je nach Genus ihre Bedeutung ändern (vgl. SERIANNI, 2015). Wie im Deutschen das Wort der/die Leiter je nach Genus unterschiedliche Bedeutungen hat, gibt es entsprechende Substantive auch im Italienischen; so hat das Nomen lama sogar drei Bedeutungen, nämlich die Klinge, das Lama und der (buddhistische) Mönch.

\section{4. Übersetzung von Märchen}

Bei jeder Übersetzung wählt der Übersetzer aus dem Repertoire einer Sprache die optimalen sprachlichen Mittel aus. Zu den wichtigsten Faktoren, die bei der Entscheidung eine Rolle spielen, gehört die Orientierung $\mathrm{m}$ potentiellen Rezipienten. Auch bei Märchen muss geklärt werden, welche Zielgruppe angesprochen werden soll, ob z.B. der Zieltext für Erwachsene als Nachweis für volkskundliche Überlieferung bestimmt ist oder für Kinder als phantasievolle Geschichte, die ihnen vor dem Schlafen vorgelesen wird. Volksmärchen sind mit verschiedenen Kultursystemen verbunden, die der Übersetzer bei seiner Arbeit berücksichtigen muss. Die Schwierigkeit des Übersetzens resultiert nicht zuletzt aus der Unterschiedlichkeit der Kultursysteme, nämlich dem der Originalsprache und dem der Zielsprache. Einen unabdingbaren Teil der Sprachkenntnisse bilden dann Realien und phraseologische Ausdrücke. Bei der Übersetzung von Nomen muss das Hauptaugenmerk vor allem auf den Artikelwörtern liegen, die die Nomen begleiten und das Genus des Substantives bestimmen. 
Anhand der drei Substantive Tod, Fuchs und Rabe (vgl. J. UND W. GRIMM 2010) soll gezeigt werden, wie der Übersetzer das Problem mit unterschiedlichen Genera gelöst hat.

\begin{tabular}{|l|l|l|}
\hline \multicolumn{1}{|c|}{ der Tod } & \multicolumn{1}{|c|}{ smrt } & \multicolumn{1}{|c|}{ la morte } \\
\hline "Wer bist du?" & "A kdopak ty jsi?" & "Chi sei?" domandò l'uomo. \\
"Ich bin der Tod, der alle gleich & "Já jsem ta, která vše srovná. & "Sono la Morte, che fa tutti \\
macht." & uguali." \\
Da sprach der Mann: & Allora l'uomo disse: \\
"Du bist der Rechte, du holst & Tu muž odvětil: & "Ano, ty jsi ta pravá, ty bereš \\
den Reichen wie den Armen & "Tu sei giusta: prendi sia il \\
ohne Unterschied, du sollst mein & chudé i bohaté bez rozdílu, ty \\
Gevattersmann sein." & bucco sia il povero senza fare \\
differenze; sarai & la mia comare." \\
\hline
\end{tabular}

Tab. 2 Der Tod in Märchen

Tabelle 2 zeigt die unterschiedliche Verwendung des Wortes Tod, das durch die Personifizierung sein Genus bei der Übersetzung wechselt. Im Deutschen ist der Tod im Unterschied zur tschechischen und italienischen Sprache männlichen Geschlechts. Dem entspricht die maskuline Bezeichnung des Todes als Gevattersmann. Da der Tod im Italienischen und Tschechischen weiblich ist, musste bei der Übersetzung und der Personifizierung das Geschlecht entsprechend berücksichtigt werden. Deswegen erscheint der Tod in den Bezeichnungen kmotrička und la comare als Frau.

Ein ähnliches Beispiel der Personifizierung findet sich in den Grimmschen Märchen beim Substantiv Fuchs, wie es Tabelle 3 verdeutlicht. In diesem Fall wird durch die im Tschechischen weibliche Form in der Übersetzung die Rolle des Tieres betont: Es wird deutlich, dass es sich um einen Fuchs des weiblichen Geschlechts, nämlich um ein Muttertier handelt, denn die Tiere treten in Märchen allgemein meistens geschlechtslos auf. Das heißt, dass es zwar in Märchen grundsätzlich nicht wichtig ist, ob es sich um ein weibliches oder männliches Tier handelt. Anders verhält es sich aber, wenn Tiere personifiziert werden und z.B. die Rolle eines Ehemannes oder einer Ehefrau übernehmen. Dabei muss der Übersetzer wieder das natürliche Geschlecht betonen, wie es im Märchen Die Hochzeit der Frau Füchsin der Fall ist. Hier wird die weibliche Form des Substantives Fuchs gebraucht, um die Geschlechtszugehörigkeit eindeutig zu klären. Im Tschechischen gibt es interessanterweise zwei Genusformen für das Substantiv Fuchs (liška, lišák), obwohl man allgemein die weibliche Form bevorzugt. Im Italienischen verwendet der Übersetzer in diesem Märchen die Bezeichnung Herr Fuchs und Frau Füchsin, um zu verdeutlichen, dass sie ein (Ehe)paar bilden. Bemerkenswert ist, dass für die männliche Form das Substantiv volpone steht, das normalerweise auch eine schlaue Person konnotiert (trifft auch für das deutsche Wort „Fuchs“ zu).

\begin{tabular}{|c|c|c|}
\hline der Fuchs (m) & Liška (f) & la volpe (f) \\
\hline $\begin{array}{l}\text { Bald darauf schlich } \\
\text { ein Fuchs vorbei, den wollten sie } \\
\text { niederschießen, aber der Fuchs } \\
\text { rief: } \\
\text { "Lieber Jäger, laß mich leben, ich } \\
\text { will dir auch zwei Junge geben." }\end{array}$ & $\begin{array}{l}\text { Za malou chvíli se jim do cesty } \\
\text { připletl lišáa. Inu když je hlad, i } \\
\text { liščí pečínka príijde k chuti. Ale } \\
\text { lišák se měl do breku: "Lovče, } \\
\text { nechej mě žít, já ti dám dva } \\
\text { mladé." }\end{array}$ & $\begin{array}{l}\text { Poco dopo, giunse } \\
\text { una volpe, volevano spararle ma } \\
\text { la volpe gridò: } \\
\text { "Caro cacciatore, se vivere potrò, } \\
\text { volentieri due piccini ti darò." }\end{array}$ \\
\hline
\end{tabular}




\begin{tabular}{|l|l|l|}
\hline \multicolumn{1}{|c|}{ der Fuchs (m) } & \multicolumn{1}{|c|}{ Liška (f) } & \multicolumn{1}{c|}{ la volpe (f) } \\
\hline $\begin{array}{l}\text { Die Hochzeit } \\
\text { der Frau Füchsin }\end{array}$ & $\begin{array}{l}\text { Kmotra liška } \\
\text { se opět vdává }\end{array}$ & La signora volpe \\
\hline
\end{tabular}

Tab. 3 Der Fuchs in Märchen

Ein interessanter Genuswechsel findet sich in dem Märchen Der Rabe, das von einem Mädchen erzählt, das in einen Raben verzaubert wurde. Bei dieser Geschichte unterscheiden sich schon die deutschen Texte. In einigen ist nur von dem Raben die Rede (vgl. J. UND W. GRIMM 2010) und in anderen wird durch die Verwendung des femininen Artikels aus dem männlichen Raben ein weiblicher, nämlich die Rabe (URL3). Die tschechische Sprache ist sehr flexibel und kann sich leicht an die Bedürfnisse des neu entstehenden Textes anpassen, wie der Okkasionalismus Havránka beweist. In der italienischen Version bleibt die männliche Form il corvo, obwohl es sich um ein weibliches Tier handelt.

\begin{tabular}{|l|c|c|}
\hline \multicolumn{1}{|c|}{ der Rabe (m) } & \multicolumn{1}{|c|}{ havran (m) } & \multicolumn{1}{|c|}{ Il corvo $(\mathbf{m})$} \\
\hline \multicolumn{1}{|c|}{ Die Rabe (f) } & Havránka (f) & Il corvo $(\mathrm{m})$ \\
\hline $\begin{array}{l}\text { Der Mann versprach alles zu } \\
\text { thun, was sie verlangt hatte. Die } \\
\text { Rabe aber sagte... }\end{array}$ & $\begin{array}{l}\text { Mládenec slíbil, že udélá vše, jak } \\
\text { si přeje, ale Havránka řekla... }\end{array}$ & $\begin{array}{l}\text { L'uomo promise che avrebbe } \\
\text { fatto tutto ciò che ella aveva } \\
\text { chiesto, ma il corvo disse... }\end{array}$ \\
\hline
\end{tabular}

Tab. 4 Der Rabe in Märchen

\section{Fazit}

In diesem Artikel werden kurz einige Aspekte der Arbeit eines Übersetzers dargestellt. Es werden Substantive erläutert, die wegen ihrer Bedeutung in der Zielsprache einen Genuswechsel erfordern.

Gerade Märchen bieten sich als geeignete Untersuchungstexte für die Genusanalyse an, weil sie viele interessante Substantive enthalten, bei denen man das Genus in der Zielsprache berücksichtigen muss. Die in den Tabellen dargestellten Ergebnisse beweisen, dass man in verschiedenen Sprachen eine adäquate Lösung finden kann, auch wenn sie zu einem Genuswechsel führt und dabei Okkasionalismen entstehen, die ohne Zweifel ihren Zauber haben können. Die Sprachen sind flexibel und bieten unglaublich viele Möglichkeiten , beim Übersetzen die Zielsprache und deren Sprachkultur zu bereichern.

Quellen

[URL1]: http://www.duden.de (20. 9. 2017).

[URL2]: https://www.korpus.cz (5. 9. 2017)

[URL3]: http://www.grimmstories.com (12. 8. 2017). 


\section{Literatur}

BERTIN, Mario (2016): Cantico delle Creature. Roma: Castelvecchi Editore.

J. UND W. GRIMM (2010): Kinder- und Hausmärchen. Stuttgard: Reclma Verlag.

HAMPLOVÁ, Sylva (2004): Mluvnice italštiny / Grammatica italiana. Praha: Leda

KONVIČKA, RAŠNEROVÁ, ZBORNÍKOVÁ (2017): Translatologické kategorie v praxi. Olomouc:

Univerzita Palackého v Olomouci

LEVÝ, Jiří (1963): Umění překladu. Praha: Academia.

REINDERS, Angela (2012): Bruder Sonne, Schwester Mond. München: Pattloch Geschenkbuch.

SERIANNI, Luca und Koll. (2015): Dizionario italiano. Milano: Garzanti.

ŠTÍCHA, František (2011): Kapitoly z české gramatiky. Praha: Academia.

WHITFIELD, John Humphreys (1986): A Short History of Italian Literature. Manchester: Manchester University Press.

Dieser Beitrag entstand mit der Unterstützung des Projekts der Schlesischen Universität in Opava SGS/4/2018 ,Analýza a interpretace textu“ / ,Text Analysis and Interpretation“.

Mgr. Markéta Šmalcová / marketa.smalcova@fpf.slu.cz

Slezská univerzita v Opavě, Filozoficko-přírodovědecká fakulta,

Ústav cizích jazyků, oddělení germanistiky, Masarykova třída 37, 74601 Opava, CZ 